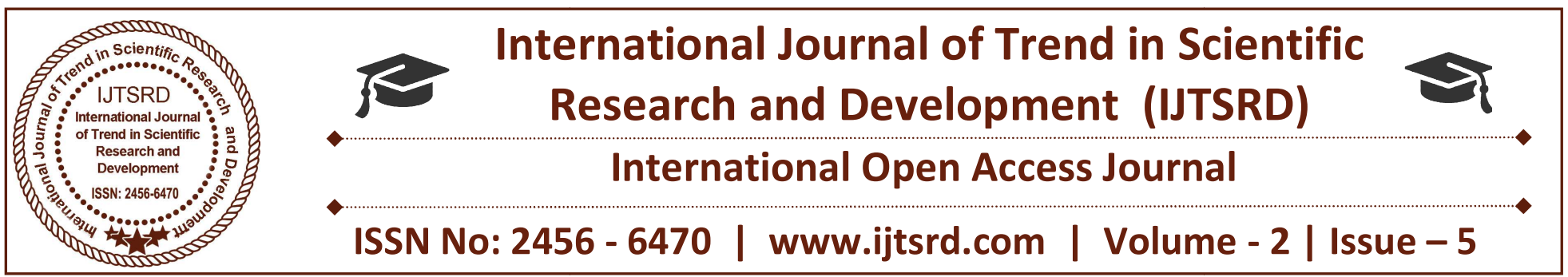

\title{
Myanmar Alphabet Recognition System Based on Artificial Neural Network
}

\author{
Myat Thida Tun \\ Lecturer, Department of Information Technology, Technological University (Thanlyin), \\ Republic of the Union of Myanmar, Banbwegon, Myanmar (Burma)
}

\begin{abstract}
This paper describes Myanmar Alphabet Recognition System Based on Neural Network. Typical pattern recognition systems are designed using two parts. The first part is a feature extractor that finds features within the data, which are specific to the task being solved. Edge detection method is used to extract image's features. It may be grouped into two categories, gradient and Laplacian. The gradient method (Roberts, Prewitt, Sobel) detects the edges by looking for the maximum and minimum in the first derivative of the image. In this paper, Sobel edge operator is chosen because it can generate the significant features for Myanmar Alphabet than other techniques. The second part is the classifier; Multilayer Perceptron Network is designed for recognition purpose. It is used to train the train data set and classify the test data set that it is shown with its result box and sound. These data sets are composed of all Myanmar alphabets. For programming and simulation of this paper, MATLAB Programming Language is used for implementation.
\end{abstract}

Keyword: Myanmar Alphabet Recognition System, Edge detection method, Multilayer Perceptron Network, MATLAB programming Language

\section{INTRODUCTION}

Character recognition is becoming more and more important in the modern world. It helps humans ease their jobs and solve more complex problems. It also is one of the popular application toolboxes. Toolboxes are comprehensive collections of MATLAB functions (M-files) that extend the MATLAB environment to solve particular classes of problem. Areas in which toolboxes are available include signal processing, control systems, neural network, fuzzy logic, wavelets, simulation, and many others Artificial Neural Networks is used to train and identify Myanmar alphabet. A Neural Networks is an information-processing paradigm that is inspired by the way biological nervous systems, such as brain, process information. There are different types of Neural Networks such as Perceptron's Network, Linear Network, Back propagation Network, Dynamic Network, Radial Basis Network, etc. Perceptron /Network is chosen for this paper. Perceptron is the simplest form of Neural Networks and function as a pattern classifier. To simulate the recognition system, MATLAB programming language is studied. In this paper, firstly, Myanmar Alphabet image files are created and saved as xx.gif file. These files are separated as train data set and test data set. Textural features: pattern, spatial frequency, homogeneity, etc. The extracted feature sets are saved as xx.txt file format. Finally, the neural networks program is applied to train files set and classify the tests data files.

\section{PROPOSED IMPLEMENTATION STEPS OF MYANMAR ALPHABET RECOGNITION}

The steps needed for the implementation of Myanmar alphabet recognition systems are described in the following articles.

\section{A. Database Creation}

Creating a database consisting of a train set and a test set. Table 1 shows file classes and numbers of train and test files. 
International Journal of Trend in Scientific Research and Development (IJTSRD) ISSN: 2456-6470

Table 1 Image Database Table

\begin{tabular}{|c|c|c|c|}
\hline $\begin{array}{c}\text { Sr. } \\
\text { No. }\end{array}$ & File Class & $\begin{array}{c}\text { No: of Train } \\
\text { File }\end{array}$ & $\begin{array}{c}\text { No: of Test } \\
\text { File }\end{array}$ \\
\hline 1 & ka. gji & 15 & 16 \\
\hline 2 & kha.gwei & 15 & 16 \\
\hline 3 & ga. nge & 15 & 16 \\
\hline 4 & ga. gji & 15 & 16 \\
\hline 5 & nga & 15 & 16 \\
\hline$\cdot$ & $\cdot$ & $\cdot$ & $\cdot$ \\
$\cdot$ & $\cdot$ & $\cdot$ & $\cdot$ \\
. &. & $\cdot$ & $\cdot$ \\
\hline 31 & ha & 15 & 16 \\
\hline 32 & la. gyi & 15 & 16 \\
\hline 33 & a & 15 & 16 \\
\hline
\end{tabular}

$\begin{array}{lllll}m & m & m & \infty & m \\ \pi & m & m & m & m \\ m & m & m & m & m\end{array}$

Figure1. Images in Ka Gji File of Train Set

\begin{tabular}{|c|c|c|}
\hline & $45,22,30,2,3,21$, & $8,12,0,1,12,14$, \\
& $21,53,15,25,35$, & $23,6,19,21,15$, \\
& $29,3,4,26,18,17$, & $1,1,22,14,18,9$, \\
& $9,45,24,21,0$, & $32,13,12,3,68$, \\
& $109,20,17,31,9$, & $8,9,18,5,3,15$, \\
& $15,22,21,5$ & 7,5 \\
\hline \multirow{6}{*}{ Sobel } & $8,20,27,56,13$, & $7,7,10,21,8,14$, \\
& $23,24,25,0,0,18$, & $1,6,0,0,11,12$, \\
& $26,26,10,61,33$, & $17,13,30,17,10$, \\
& $37,7,8,25,12,51$, & $5,6,20,14,38$, \\
& $6,22,30,15,0$, & $12,12,12,18,0$, \\
& $115,19,22,8,11$, & $71,7,7,12,6,19$, \\
& $38,19,24,9$ & $10,10,4$ \\
\hline
\end{tabular}

Sobel operator is shown in Figure 2.Neural Network Program executes the classification of images on the basis of the set of features have been extracted. This program applies Multilayer Perceptron Network because it is the simplest method for classification. The hard limit transfer function in this method is used to set the output and create neurons that classify inputs into two distinct categories. Figure 3 shows the flowchart of Neural Network Program.

\section{B. Programming Parts}

There are two program parts in Software Implementation for Myanmar Alphabet Recognition System: feature extraction and Neural Networks programs. These programs are written in $\mathrm{M}$-file of MATLAB. The user must be prepared a database consisting of train, test and target files before the programs start in MATLAB command window. In feature extraction portion, Robert, Prettwit and Sobel operators are testd to detect edges of images. By comparing the extracted features, Sobel operator is found that it can generate the most significant features for each alphabet. Table 2 shows the example of extracted features which are grouped in every 5 degree bins for ka. gji and ga. nge alphabets. Based on these facts, sobel operator is selected in feature extraction program.

Table 2 Types of Operators and Features of Images

\begin{tabular}{|c|c|c|}
\hline \multirow{2}{*}{ Operators } & \multicolumn{2}{|c|}{ Images } \\
\cline { 2 - 3 } & $\mathbf{k a}$ gii & ga. nge \\
\hline \multirow{5}{*}{ Robert } & $0,0,6,21,5,20$, & $0,0,4,4,1,9,4$, \\
& $17,4,0,0,7,14$, & $4,0,0,4,48,3$, \\
& $17,1,11,9,0,0,0$, & $4,1,0,0,0,0,7$, \\
& $0,7,12,2,13,16$, & $7,3,9,12,2,0$, \\
& $7,0,299,5,13,30$, & $245,2,10,20,5$, \\
& $6,13,6,0,0$ & $12,2,0,0$ \\
\hline Prewitt & $3,26,28,20,14$, & $4,13,7,14,3,22$, \\
\hline
\end{tabular}

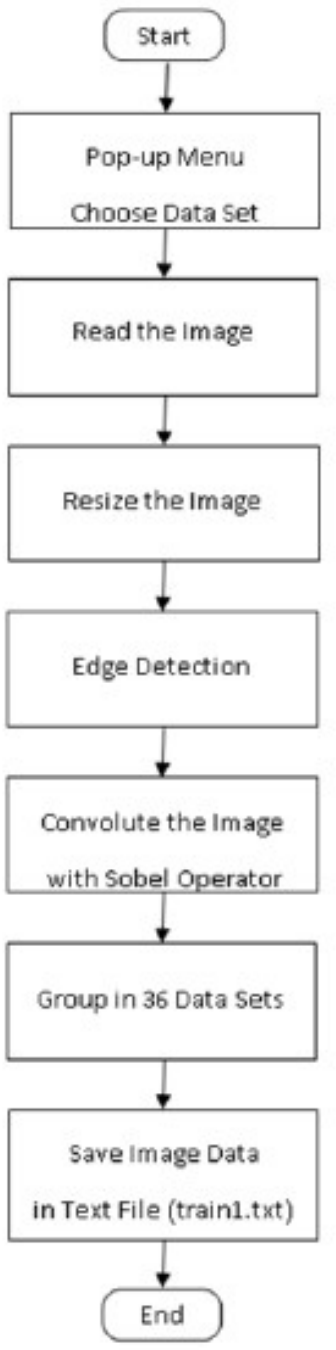

Figure2. Flowchart for Feature Extraction 


\section{III.EXPERIMENTAL PARAMETERS}

The purpose of this program is to extract features of images in train and test files. When this program runs, a menu box appears asking the user whether the user want to run the algorithm on test or train sets. If the user chooses train set, the second menu will appears for the user to choose the type of character files. These menu boxes are shown in Figure 4.

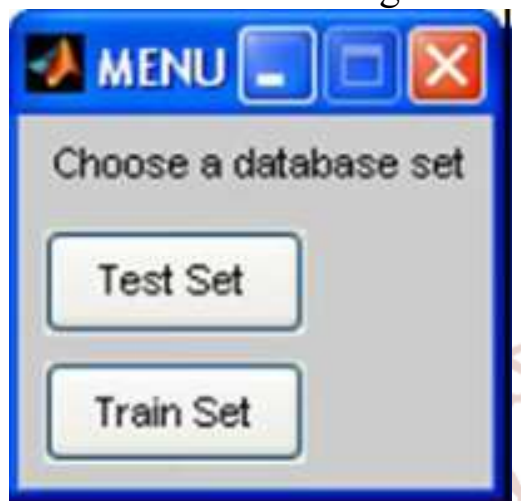

(b)

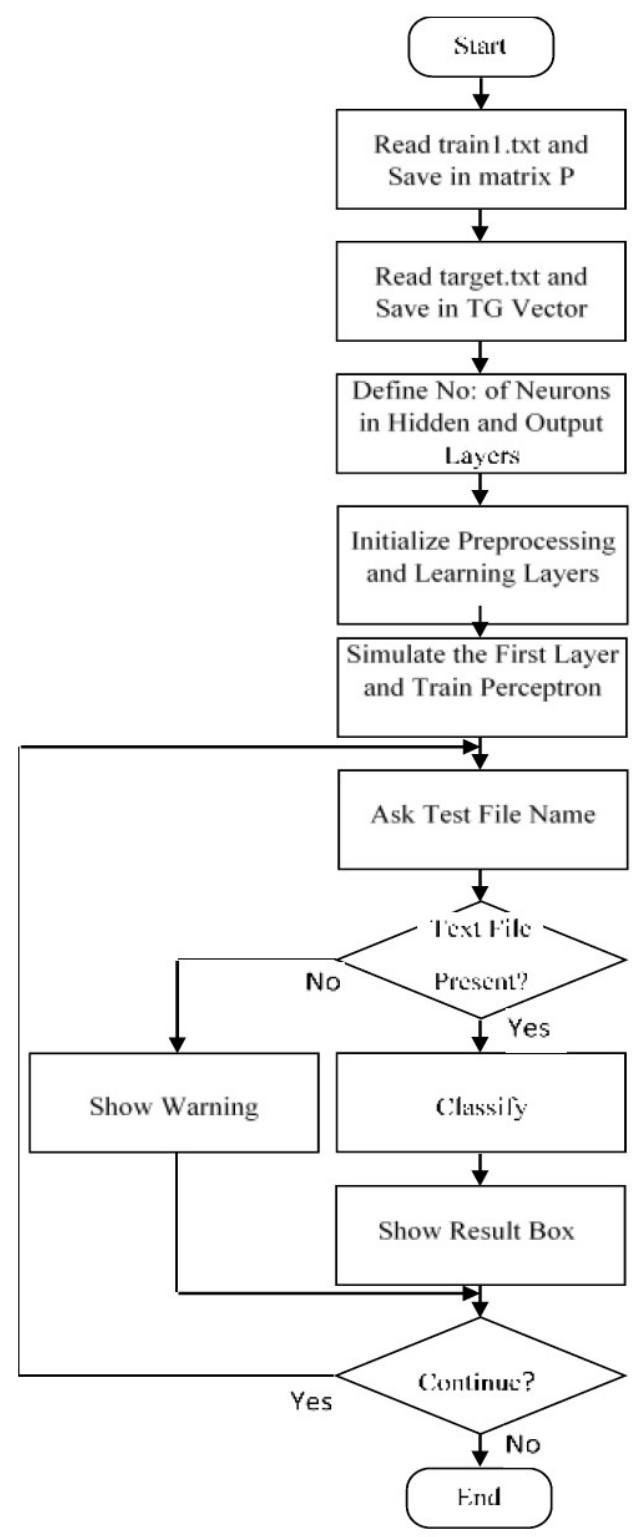

Figure3. Flowchart for Neural Network Program

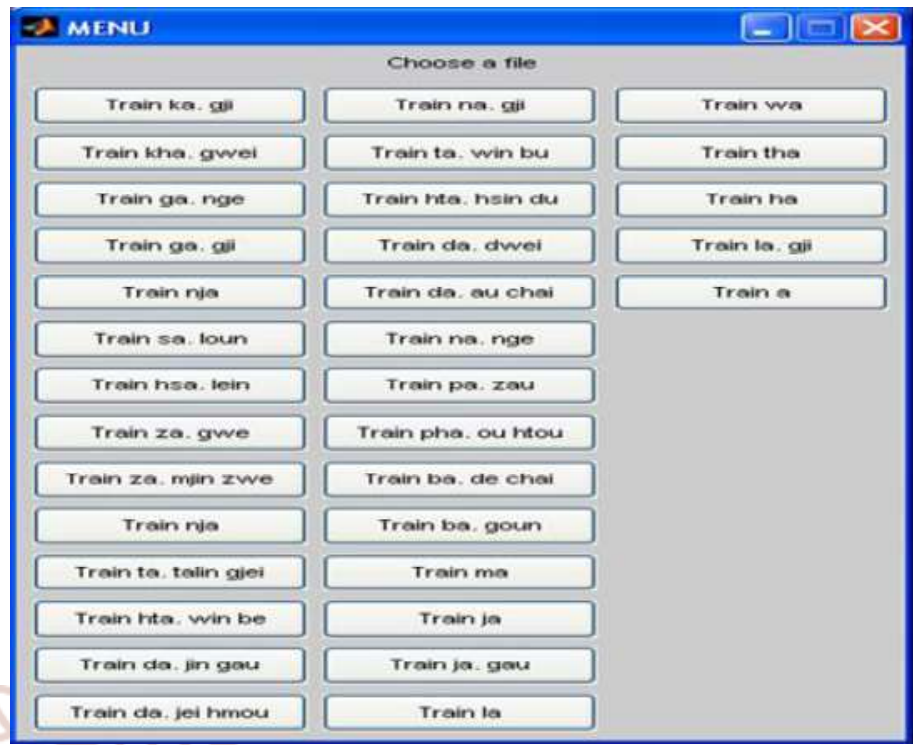

(a)

Figure4. Pop-up Menus: (a) Image File Menu and (b) Database Set Menu

One type of character files is chosen and then the user must input the numbers of images in train file. A for loop is used to read an entire file of images as strings and store them in MATLAB's memory. The image string is resized to $150 \times 140$ pixels. This size is the optimal for enough detail while keeping the processing time low. The original image and resized image of ka. gji are shown in Figure 5.

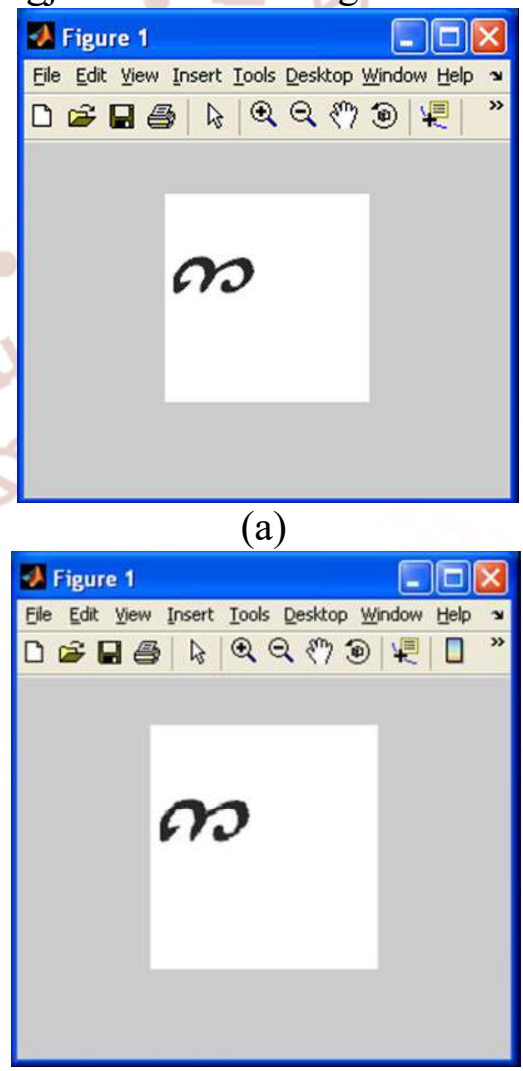

(b)

Figure5. Images: (a) Original Image and (b) Resize Image 
The next step is to detect the edges of images using Sobel operator. The images and this operator are convoluted in $\mathrm{x}$ - and $\mathrm{y}$-directions, and the gradient of images is estimated. Then, the direction of gradient is calculated and the resulting matrix is rearranged into a column vector by using im2col MATLAB function. Finally, the column is converted from the radians to the degrees. The orientation magnitude is read every 5 degrees and summed to form a bin for the orientation histograms. The numbers of histogram bin are 36 bins because the actual resolution of each bin is set to 5 degree. These bins are stored in train 1.txt file to feed to the Neural Network. If the user chooses test set, the set but the features of test set is automatic stored in text file. MATLAB Programming develops this algorithm that is feature extraction method.

\section{IV.EXPERIMENTAL RESULT}

Neural Network Programming Steps: There are many steps in Neural Network program. If the user wants to run this program, the following command has to type in command window of MATLAB.

$>>$ NN-Program At this time, cover window appears as shown in Figure 6.

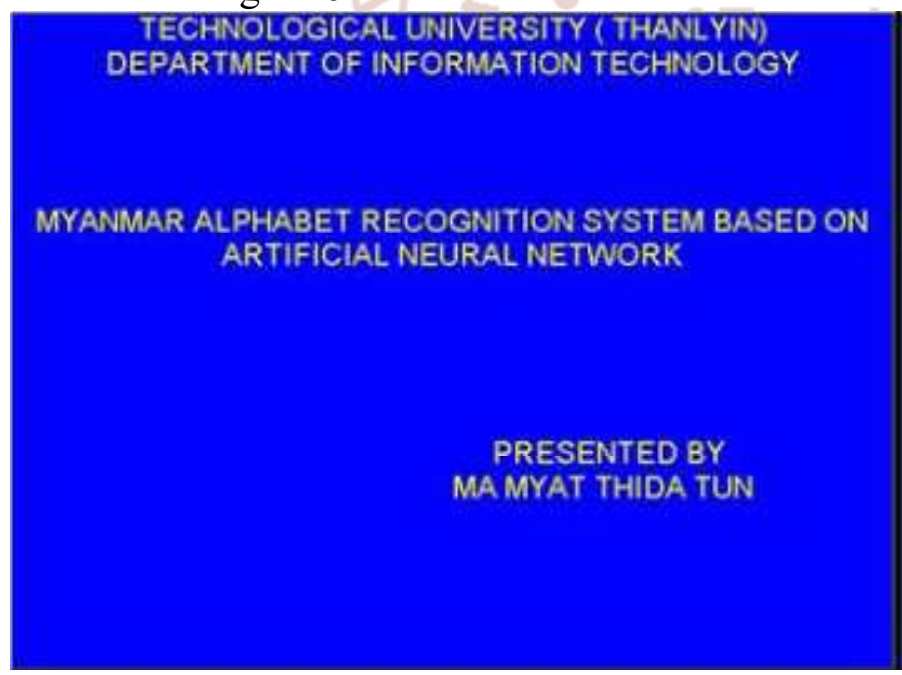

Figure6. Cover Window

When the training process starts, the plot of sumsquare error and epoch shown in Figure 7 can be seen. When the sum-square error reaches to zero or the training reach to the defined epochs as shown in Figure 8, the next processes can be performed.

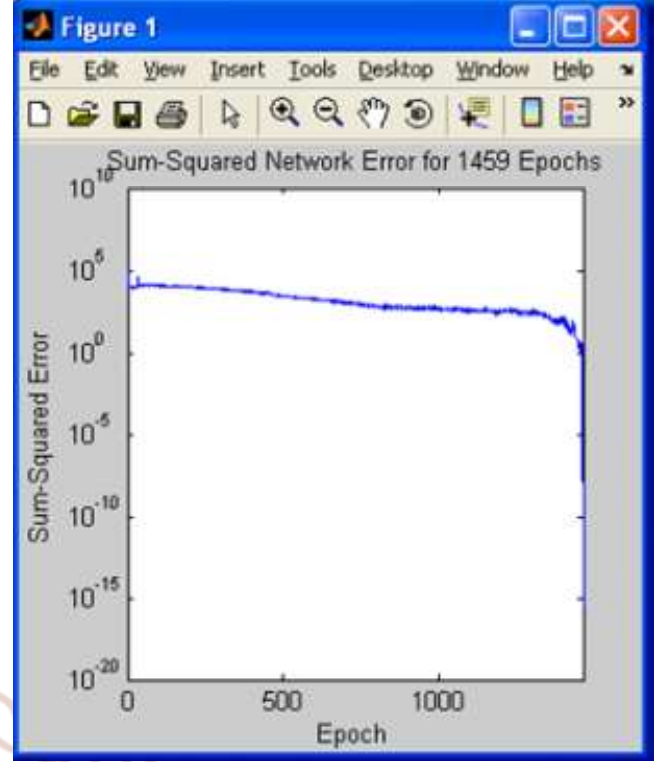

Figure7. The Plot of Sum-square Error and

\section{Epoch}

After the training process, program asks the user to enter the test file name to be classified. If the user writes the invalid test file name, warning message box will appear as shown in Figure 9.
Figure8. End of Training Neural Network

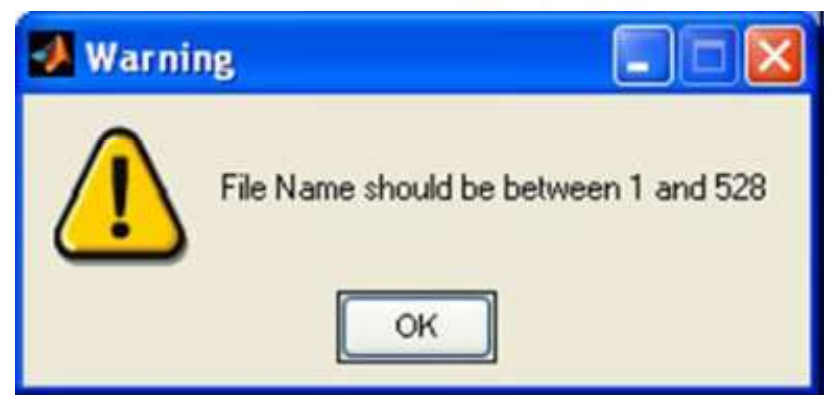

Figure9. Warning Message Box 
When the test file is in the valid range, the Multilayer Perceptron network classifies this file and the original image with the result box will appear. The classified image, ka. gji, and its result box are shown in Figure 10 .

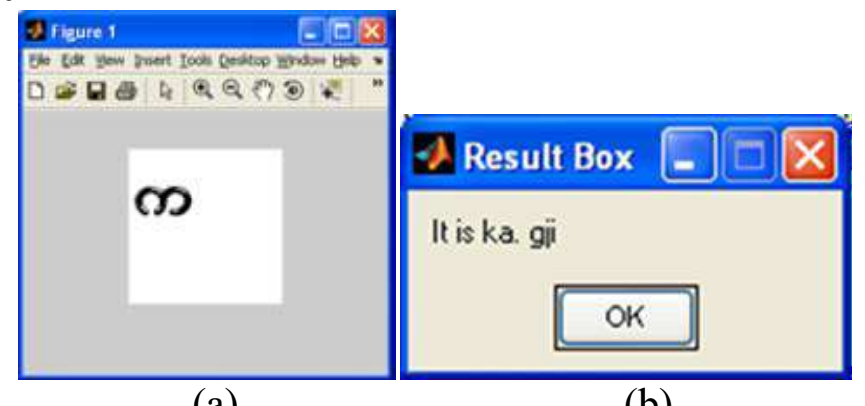

(a)

Else, the testing process reaches to the end of pattern recognition that can be viewed with the message box as Figure 13.

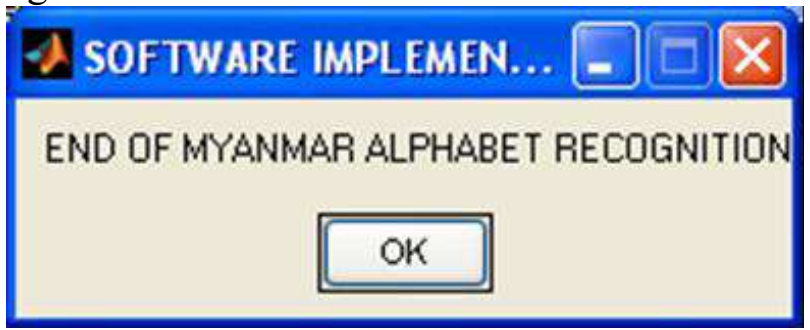

Figure13. Message Box of Ending Program

\section{CONCLUSIONS}

In this paper, pattern recognition systems are designed using two parts. The first part is a feature extractor that finds features within the data which are specific to the task being solved (finding pixels within an image for character recognition). When the feature extract program is tested with various operators, Sobel edge operator is found that it is the best one for Myanmar Alphabet Recognition System. Therefore, this operator is chosen. The performance of recognition can be increased when the input training data files are increased. The size of database set is present as an important role of the training section. For the second part, classification, the Multilayer Perceptron Neural Network is discussed and implemented in this paper. It can also be used for almost any image recognition applications. The network is a 3 layer configuration, with the required input and output layers, as well as a single hidden layer that choosing the number of hidden layers is a difficult task with no hard rules or guidelines. However, the size of a hidden layer is related to the features or distinguishing characteristics that are to be discerned from the data. Here, the network applies one hidden layer, which has 300 neurons. The output layer is where the output vector can be retrieved. Each neuron in this layer outputs a value between 0 and 1 , which is guaranteed by the use of a hard limit transfer function when calculating each neuron's output.

\section{REFERENCES}

1. Anon., "Neural Networks", Stat Soft, Inc., 2006.

2. ShwetaYewale,"NeuralNetworkToolbox5.0.1", $<$ ht tp://www.mathworks.com/products/neuralnet/desc riptio2.html>, 2006.

3. Pankaj K. Bharne "Artificial Neural Network Approach For Han Gesture Recognition", International Journal of Engineering Science and Technology (IJEST), vol. 3(4), pp. 2603- 2608, . (2011, Apr). 
4. Ankit Chaudhary, J. L. Raheja, Karen Das, and Sonia Raheja "Intelligent Approaches to interact with Machines using Hand Gesture Recognition in Natural way A Survey," International Journal of Computer Science \& Engineering Survey (IJCSES), vol. 2, . (2011, Feb).

5. Pradeep, J. et al.-Diagonal based feature extraction for handwritten alphabet recognition system using neural network, International Journal of Computer Science and Information Technology, Vol.3, No.1, pp. 27, 2011

6. Khurana,P., Singh, V. A model for human cognition,\| International Journal of Computing and Business Research, Vol.2, Issue.3, 2011

7. Kurian, C, Balakriahnan, K, Continuous speech recognition system for Malayalam language using PLP cepstral coefficient, J Journal of Computing and Business Research, Vol.3, Issue.1, 2012

8. Sivanandam, S. N, Deepa, S.N., Principle s of Soft Computing,\| Wiley-India publisher, 2nd edition, ISBN: 978-81-265-2741-0, 2011

9. Proceeding of 8th International Symposium on Intelligent Systems and Informatics (SISY), pp.249-251, 2010

10. Dash, T., "Time Efficient Approach to Offline Hand Written Character Recognition using Associative Memory Net"l, International Journal of Computing and Business Research, ISSN: 2229 -6166, Vol. 3, Issue-3, 2012.

11. Dash, T., Chattopadhyay, S. Nayak T., Handwritten Signature Verification using Adaptive Resonance Theory Type-2 (ART-2) Netll. Journal of Global Research in Computer Science, vol. 3, issue 8, pp. 2125, 2012

12. Dash, T., Nayak, T., Chattopadhyay, S., -Offline Verification of Hand Written Signature Using Adaptive Resonance Theory Net (Type-1)\|, in Proc: IEEE Int. Conf. Electronics Computer Technology (ICECT), Vol-2, pp. 205-210, 2012.
13. Ravina Mithe, Supriya Indalkar, Nilam Divekar "Optical Character Recognition" International Journal of Recent Technology and Engineering (IJRTE) ISSN: 2277-3878,Volume-2, Issue-1, March 2013

14. Parappa S.N. and Singh M.P., "Performance Analysis of Conjugate Descent Learning Rulof Feed Forward Neural Networks for Pattern Classification", International Journal of Multidisciplinary and Current Research, pg. 723725, Vol. 3, 2015

15. Kumar Prodhan, Md. Farukuzzaman Khan "Implementation of Back-Propagatio Neural Network for Isolated Bangla Speec Recognition" International Journal of Information Sciences and Techniques (IJIST)Vol.3, No.4, July 2013

16. Farjana Yeasmin Omee, Shiam Shabbir Himel "A Complete Workflow for Development of Bangla OCR" International Journal of Computer Applications (0975 - 8887) Volume 21- No.9, May 2011

17. Md. Abul hasnat "Research Report on Bangla OCR training and testing method" Conference on Artificial Intelligence and Soft Computing, Marlbella Spain, ACTA press 2004.

18. GOLABI, S.SAADAT, S- HELFROUSH, M. S.TASHK, A.: A novel thinning algorithm with fingerprint minutiae extraction capability, International Journal of Computer Theory and Engineering 4 (2012),no. 4, 514-517.

19. E. Robust feature extraction in fingerprint images using ridge model tracking, Center for Embedded Systems for Critical Applications, Bradley Department of Electrical and Computer Engineering (2014).

20. SUANDI, S.A.: Finger Code for identity verification using fingerprint and smart card in: 10th Asian Control Conference (2015), 6p.em_Classification_UsingTransformation_Base d_Feature_Extraction_Methods [accessed Jul 07 2018] 